\title{
Interférences
}

Ars scribendi

\section{Cicéron entre histoire et biographie}

Propositions pour une relecture de la lettre de Cicéron à Lucceius (ad Fam. 5, 12)

\section{Gérard Salamon}

\section{(2) OpenEdition \\ 12 Journals}

Édition électronique

URL : http://journals.openedition.org/interferences/899

DOI : 10.4000/interferences.899

ISSN : $1777-5485$

Éditeur

HiSoMA - Histoire et sources des Mondes antiques

Édition imprimée

Date de publication : 1 janvier 2009

ISSN : 1777-5485

\section{Référence électronique}

Gérard Salamon, «Cicéron entre histoire et biographie », Interférences [En ligne], 5 | 2009, mis en ligne le 11 décembre 2014, consulté le 15 septembre 2020. URL : http://journals.openedition.org/ interferences/899; DOI : https://doi.org/10.4000/interferences.899

Ce document a été généré automatiquement le 15 septembre 2020.

Tous droits réservés 


\section{Cicéron entre histoire et biographie}

Propositions pour une relecture de la lettre de Cicéron à Lucceius (ad

Fam. 5, 12)

\section{Gérard Salamon}

1 L'ouvrage bien connu que François Hartog et Michel Casevitz ont consacré à l'histoire antique ${ }^{1}$ présente en tout et pour tout deux textes de Cicéron : la fameuse digression sur l'histoire ${ }^{2}$ et la lettre à L. Lucceius ${ }^{3}$, avec pour celle-ci le commentaire suivant: "Après la théorie (scil.le de Oratore), la fabrique ou la cuisine de l'histoire contemporaine ${ }^{4} »$. Une telle présentation, en dehors même des problèmes chronologiques qu'elle pose ${ }^{5}$, est tout à fait caractéristique de la lecture qu'un certain nombre de critiques font de la lettre à Lucceius. Elle serait révélatrice du double langage que tient Cicéron lorsqu'il traite de l'histoire, énonçant dans ses traités rhétoriques de grands et beaux principes sur le respect par l'historien de la vérité ${ }^{6}$ et s'empressant de recommander à Lucceius de les oublier lorsqu'il lui demande d'écrire l'histoire de son consulat ${ }^{7}$. Ce à quoi les défenseurs de l'orateur rétorquent en substance que, l'ouvrage demandé à Lucceius relevant exclusivement de la stratégie de propagande développée par Cicéron pour rétablir son influence à son retour d'exil ${ }^{8}$, on ne peut utiliser sa lettre pour définir la conception qu'il a de l'histoire 9. Mais il n'est pas certain que, dans un cas comme dans l'autre, le problème soit bien posé : c'est la raison pour laquelle j'aimerais reprendre ici l'étude de la lettre à Lucceius autour d'une question qui est, me semble-t-il, beaucoup moins simple qu'il n'y paraît : à quel genre littéraire l'œuvre que Cicéron demande à Lucceius d'écrire appartient-elle exactement? Mais, avant d'aborder la lettre elle-même, je voudrais rappeler en quelques mots, car la chose n'est pas sans importance, ce que nous savons de L. Lucceius et de ses relations avec Cicéron ${ }^{10}$.

2 Même s'il a eu une carrière politique moins brillante que celle de Cicéron, L. Lucceius ${ }^{11}$ a joué un rôle non négligeable dans les affaires de son époque : préteur, sans doute en $67^{12}$, il est connu pour avoir fait cesser en 64 les poursuites lancées par César contre les partisans de Sylla qui avaient assassiné des proscrits en accusant Catilina pour le même motif; il fait ensuite partie des soutiens de Cicéron lors de son consulat ${ }^{13}$. Candidat à son tour au consulat pour l'année 59 après avoir passé un accord avec César, 
il est finalement battu par Bibulus soutenu par les optimates ${ }^{14}$. En 49 , il apparaît, selon César lui-même, comme l'un des plus proches conseillers de Pompée ${ }^{15}$, ce qui ne l'empêche pas d'obtenir son pardon après Pharsale : c'est de Rome qu'il écrit à Cicéron en 45 , peu après la mort de Tullia ${ }^{16}$. Il est possible, même si nous n'avons aucune certitude sur ce point, qu'il ait finalement été l'une des victimes des proscriptions de $43^{17}$.

3 L. Lucceius est donc un membre éminent de l'aristocratie romaine, auquel Cicéron reconnaît en 56 ou 55, dans la lettre qui nous occupe, "l'autorité d'un homme de grande réputation et de haut rang, qui s'est fait tout particulièrement connaitre et apprécier dans les circonstances les plus dramatiques pour l'État ${ }^{18}$ ». C'est au titre de son expérience des affaires publiques que, conformément aux habitudes romaines, L. Lucceius se consacre à l'histoire. En un mot, comme l'a récemment souligné Jon Hall dans un article très éclairant ${ }^{19}$, Lucceius n'est ni Archias ni Thyillus, auxquels Cicéron avait demandé une œuvre poétique sur son consulat ${ }^{20}$; ce n'est pas non plus Posidonius auquel il avait envoyé à Rhodes, pour qu'il le mette en forme, un mémento en grec qu'il avait lui-même écrit sur le sujet ${ }^{21}$. On peut certes s'interroger sur la profondeur réelle des liens qui unissent les deux hommes ${ }^{22}$, mais Lucceius est pour l'orateur l'un de ses pairs et c'est dans ce contexte qu'il faut étudier la demande qu'il lui adresse. Mais que lui demande-t-il au juste?

4 À première vue, la réponse à cette question est évidente : Cicéron aimerait obtenir que Lucceius, qui est engagé dans la rédaction d'une œuvre historique continue de grande ampleur ${ }^{23}$ dans laquelle les termes de la lettre invitent à reconnaître des Annales ${ }^{24}$, interrompe un moment le cours de son travail pour consacrer à la conjuration de Catilina et au rôle qu'il y a joué une monographie :

Sed quia uidebam Italici belli et ciuilis historiam iam a te paene esse perfectam, dixeras autem mihi te reliquas res ordiri, deesse mihi nolui quin te admonerem ut cogitares coniunctene malles cum reliquis rebus nostra contexere an, ut multi Graeci fecerunt, Callisthenes Phocicum bellum, Timaeus Pyrrhi, Polybius Numantinum, qui omnes a perpetuis suis historiis ea quae dixi bella separauerunt, tu quoque item ciuilem coniurationem ab hostilibus externisque bellis seiungeres ${ }^{25}$.

5 Les limites temporelles de cette monographie sont précisées plus loin lorsque Cicéron vante à son correspondant l'intérêt et les avantages du projet :

Quod si te adducemus ut hoc suscipias, erit, ut mihi persuadeo, materies digna facultate et copia tua. A principio enim coniurationis usque ad reditum nostrum uidetur mihi modicum quoddam corpus confici posse ${ }^{26}$...

6 Il s'agirait donc pour Lucceius de traiter la période qui va de 63 - si l'on fixe bien en cette année-là le début de la conjuration, ce qui peut se discuter ${ }^{27}$ - à 57 ; et, au moins en première analyse, l'ouvrage concerné paraît bien relever de l'histoire : c'est ce que l'on conclut des exemples donnés par Cicéron, qui renvoie Lucceius aux monographies rédigées par Callisthène, Timée et Polybe, même si l'on peut considérer que la comparaison est quelque peu forcée puisque les monographies grecques citées étaient consacrées non pas à des conflits internes mais à des guerres et au moins pour Timée et Polybe à des guerres extérieures ${ }^{28}$.

7 Mais on notera que Cicéron, qui connaît le sens des mots, n'utilise jamais dans sa lettre les termes historia ou historiae pour définir la monographie à laquelle il voudrait que se consacre Lucceius : historia, qui apparait à quatre reprises (deux fois au singulier, deux fois au pluriel), désigne en revanche soit les "Histoires générales» écrites par Callisthène, Timée et Polybe par opposition à leurs monographies ${ }^{29}$, soit l'histoire en 
tant que genre, avec ses lois ${ }^{30}$, soit enfin, à deux reprises, l'œuvre historique que Lucceius est en train d'écrire ${ }^{31}$. Ces deux dernières occurrences sont particulièrement significatives parce que Cicéron y évoque parallèlement l'ouvrage qui fait l'objet de sa lettre. La première fois, il se contente pour désigner celui-ci d'un très vague nostra ${ }^{32}$; la seconde fois, il est infiniment plus explicite :

Quo mihi acciderit optatius si in hac sententia fueris, ut a continentibus tuis scriptis, in quibus perpetuam rerum gestarum historiam complecteris, secernas hanc quasi fabulam rerum euentorumque nostrorum. Habet enim uarios actus multasque mutationes et consiliorum et temporum ${ }^{33}$.

Il faut bien entendu tenir compte, dans l'opposition entre perpetuam rerum gestarum historiam et hanc quasi fabulam rerum euentorumque nostrorum de la limitation introduite par Cicéron (hanc quasi fabulam); mais la référence à la tragédie est claire, d'autant que le terme fabula est expliqué (enim) par la formule habet enim uarios actus multasque mutationes et consiliorum et temporum ${ }^{34}$. Par ailleurs, le terme fabula est lui-même comme anticipé par la manière dont, au début de la lettre, Cicéron présente à Lucceius les avantages d'une monographie :

Et simul, si uno in argumento unaque in persona mens tua tota uersabitur, cerno iam animo quanto omnia uberiora atque ornatiora futura sint ${ }^{35}$.

9 Les termes argumentum («sujet» ou «argument» d'une pièce) et persona («personnage ») renvoient le lecteur à l'univers du théâtre, de même que le verbe ornare, que Cicéron emploie certes couramment dans ses traités rhétoriques à propos du style ${ }^{36}$, mais dont on sait qu'il signifie aussi « costumer » dans la langue du théâtre 37 .

10 On a cherché à expliquer le passage qui s'opère ici de l'histoire à la tragédie par l'influence sur la pensée de Cicéron de l'historiographie hellénistique, qui n'est de fait pas avare en matière d'effets dramatiques ${ }^{38}$. Mais à Rome même, histoire et tragédie ont toujours entretenu des liens étroits comme en témoignent les tragédies prétextes d'époque républicaine dont nous connaissons les titres et quelques fragments, à défaut d'avoir conservé les pièces elles-mêmes ${ }^{39}$; la tragédie que, selon Asinius Pollion, L. Cornelius Balbus avait, en 43 , fait représenter à Gadès ${ }^{40}$ ainsi que, au $\mathrm{I}^{\mathrm{er}}$ siècle apr. J.-C, l'Octavie illustrent la permanence du genre. De ce point de vue, la référence à Naevius que l'on trouve ensuite ${ }^{41}$ n'est donc pas seulement, de la part de Cicéron, une citation érudite; elle participe au contraire pleinement du rapprochement qui s'opère ici entre histoire et tragédie.

11 Une telle présentation, dans une lettre à un historien, n'est évidemment pas sans conséquence : elle revient à mettre au premier plan ce que l'on pourrait appeler «les acteurs de l'histoire » et non plus les événements historiques eux-mêmes, donc à passer d'une écriture de type historique à une écriture de type biographique. Cicéron justifie ce déplacement, qui est au sens strict un changement de point de vue, par l'intérêt que manifestent les lecteurs pour la destinée des grands hommes alors qu'ils en éprouvent fort peu pour les récits annalistiques ${ }^{42}$. Mais, de manière plus prosaïque, on voit surtout l'avantage qu'il peut en retirer pour son propre compte: comme l'explique Polybe dans un passage célèbre, le genre biographique, qu'il a lui-même pratiqué en écrivant une Vie de Philopoemen, autorise en effet «l'amplification des faits » que l'histoire interdit, car biographie et éloge sont liés ${ }^{43}$. Or, de façon très significative, le champ lexical de l'éloge - celui des grands hommes, de Cicéron ou de Lucceius -, organisé autour des termes laus, laudare, celebrare, illustrare et ornare, envahit 
littéralement l'espace de la lettre ${ }^{44}$. Il est parfaitement clair que c'est moins une «histoire de son consulat» qu'un «éloge de son action» que Cicéron demande à Lucceius.

12 Mais si l'on accepte cette analyse, il faut, me semble-t-il, prendre au sérieux la demande faite par Cicéron à Lucceius d'oublier un moment les lois de l'histoire :

Itaque te plane etiam atque etiam rogo ut et ornes ea uehementius etiam quam fortasse sentis et in eo leges historiae neglegas gratiamque illam de qua suauissime quodam in prohoemio scripsisti, a qua te flecti non magis potuisse demonstras quam Herculem Xenophontium illum a Voluptate, eam, si me tibi uehementius commendabit, ne aspernere amorique nostro plusculum etiam quam concedet ueritas largiare ${ }^{45}$.

Il ne s'agit évidemment pas de demander à Lucceius de revenir sur les principes qu'il a pris soin d'édicter dans la préface historique que Cicéron cite: une telle demande, même faite avec humour, tiendrait de la provocation envers un historien qui a fait de l'impartialité sa " marque de fabrique ", ce qui serait à coup sûr contre-productif si l'on tient compte des relations, que j'ai rappelées plus haut, entre les deux personnages. Il s'agit de lui suggérer de s'illustrer dans un autre genre, celui de l'éloge, qui relève par nature de la biographie plus que de l'histoire et qui obéit donc à d'autres «lois », celles qui se trouvent précisément définies ici: gratiam... ne aspernere amorique nostro plusculum etiam quam concedet ueritas largiare. Cicéron prend même soin de donner à Lucceius un exemple parfaitement clair, pour un historien comme lui, de ce que pourrait être l'œuvre qu'il espère lui voir écrire en citant, même si la référence est un peu forcée ${ }^{46}$, l'Agésilas de Xénophon:

Nec minus est Spa<r>tiates Agesilaus ille perhibendus, qui neque pictam neque fictam [tam] imaginem suam passus est esse, quam qui in eo genere laborarunt. Vnus enim Xenophontis libellus in eo rege laudando facile omnis imagines omnium statuasque superauit ${ }^{47}$.

Mais - et c'est sans doute un des aspects les plus intéressants du problème que pose la lettre à Lucceius - Cicéron, tout en distinguant pour des raisons évidentes l'histoire et la biographie à fonction élogieuse, doit prouver à Lucceius que les deux genres sont suffisamment proches pour qu'un historien puisse se consacrer à l'un et à l'autre. C'est la leçon qu'il a lui-même tirée de ses connaissances en matière d'historiographie grecque ${ }^{48}$ et en l'occurrence les faits lui donnent raison ${ }^{49}$. Mais de ce point de vue, l'exemple de Xénophon est particulièrement intéressant. Il est évidemment impossible de savoir si Cicéron connaissait assez l'œuvre de l'historien grec pour avoir noté que le matériau de l'Agésilas provenait en grande partie des Helléniques et que Xénophon l'avait simplement réorganisé en le recentrant sur la personnalité d'Agésilas pour faire de lui le champion de la cause panhellénique ${ }^{50}$. Mais en tout état de cause, l'historien grec - que Lucceius mentionnait lui-même, comme la lettre de Cicéron nous l'apprend, dans une des préfaces de son œuvre historique et que Cicéron cite dans le de Oratore comme l'un des maîtres de l'histoire grecque ${ }^{51}$ - avait l'avantage de constituer, pour convaincre Lucceius, le précédent incontestable d'un historien qui avait pratiqué à la fois l'histoire et la biographie.

15 Il ne faut donc pas s'étonner que Cicéron, après avoir demandé à Lucceius de rédiger une œuvre dans laquelle il oublierait (pour un moment) les lois de l'histoire, lui explique que cette œuvre lui permettrait de donner toute la mesure non seulement de son talent littéraire mais aussi de ses qualités d'historien:

A principio enim coniurationis usque ad reditum nostrum uidetur mihi modicum quoddam corpus confici posse, in quo et illa poteris uti ciuilium commutationum scientia uel in explicandis causis rerum nouarum uel in remediis incommodorum, cum et reprehendes ea 
quae uituperanda duces et quae placebunt exponendis rationibus comprobabis et, si liberius, ut consuesti, agendum putabis, multorum in nos perfidiam, insidias, proditionem notabis. Multam etiam casus nostri uarietatem tibi in scribendo suppeditabunt plenam cuiusdam uoluptatis, quae uehementer animos hominum in legendo te scriptore tenere possit ${ }^{52}$. s'adresse à Lucceius pour faire l'éloge de son action, il suffit de rapprocher ces lignes du passage du de Oratore dans lequel Antoine énumère, à la fin de sa digression, les obligations de ceux qui veulent écrire l'histoire: il souligne d'abord qu'ils doivent « exposer les causes» (explicare causas) des événements pour les expliquer et porter un jugement sur les décisions qui ont été prises, mais également, ce qui n'est pas moins significatif, qu'ils doivent parler non seulement des actes (res gestae) des hommes qui comptent (qui fama ac nomine excellunt) mais aussi de leur vie et de leur caractère (cuiusque uita atque natura) ${ }^{53}$.

C'est la raison pour laquelle je ne pense pas que ce soit par pure flagornerie que Cicéron insiste sur le fait qu'en écrivant l'œuvre qu'il lui demande Lucceius immortaliserait aussi son propre nom :

Neque autem ego sum ita demens ut me sempiternae gloriae per eum commendari uelim qui non ipse quoque in me commendando propriam ingeni gloriam consequatur ${ }^{54}$.

Il est parfaitement possible, me semble-t-il, que Cicéron pense réellement que s'illustrer dans la biographie par l'éloge qu'il ferait de lui, permettrait à Lucceius d'égaler, voire de dépasser, les historiens grecs qu'il lui donne comme exemple.

Il ne faut donc pas, à mon sens, sous-estimer l'importance de la lettre à Lucceius dans la réflexion cicéronienne sur l'histoire. Que Cicéron cherche à obtenir de Lucceius une œuvre qui serve à sa propagande, cela va sans dire. Qu'on moque les prétentions de l'orateur à s'identifier à Thémistocle, à Agésilas ou à Timoléon ou que l'on critique son désir de voir célébrer sa gloire de son vivant, pourquoi pas ${ }^{55}$ ? Il n'en reste pas moins que sa lettre met en lumière les liens étroits qui existent pour les Anciens, comme en témoigne l'historiographie grecque, entre l'histoire et au moins une certaine forme de biographie, celle qui relève de l'éloge. De ce point de vue, la position exprimée ici par Cicéron - les deux genres obéissent à des lois différentes mais demandent les mêmes qualités de la part de ceux qui voudraient les pratiquer - invite à reconsidérer les questions génériques : elle revient en effet à faire de la biographie peut-être moins un genre littéraire à part que l'une des modalités de l'histoire.

\section{BIBLIOGRAPHIE}

\section{Textes anciens}

Cicero's Letters to Atticus, éd. D.R. Shackleton Bailey, 1961.

Cicero, Letters to Friends, éd. D.R. Shackleton Bailey, The Loeb Classical Library 205, 216, 230 ,

Cambridge (Mass.) - Londres, 2001, 3 vol.

Interférences, 5 | 2009 
Cicéron, Correspondance, II-III, éd. L.-A. Constans, CUF, Paris, 1936.

Xénophon, Constitution des Lacédémoniens ; Agésilas ; Hiéron ; suivi de Pseudo-Xénophon, Constitution des Athéniens, éd. M. Casevitz, 2008.

\section{Textes modernes}

BARDON H. 1952, La littérature latine inconnue. I, L'époque républicaine, Paris.

BENFERHAT Y. 2005, Dictionnaire des philosophes antiques, IV, éd. R. Goulet, Paris, s.v. « L. Lucceius », p. 130-131.

BROUGHTON T.R.S. 1986, The Magistrates of the Roman Republic. III, Supplement, Philological

Monographs 15, Atlanta (Ga.).

DENIAUX É. 1993, Clientèles et pouvoir à l'époque de Cicéron, Coll. de l'École française de Rome 182,

Rome.

HALL J. 1998, « Cicero to Lucceius (Fam. 5, 12) in its social Context: ualde bella? », CPh 93, p. 308-321.

HARTOG F., CASEVITZ M. 1999, L'histoire d'Homère à Augustin, Points. Série Essais 388, Paris.

HINARD F. 1985, Les proscriptions de la Rome républicaine, Coll. de l'École française de Rome 83, Rome.

JENKINSON E. 1967, « Nepos - An introduction to Latin Biography », in T.A. Dorey (éd.), Latin

Biography, Studies in Latin Literature and Its Influence, Londres, p. 1-15.

MANDEL J. 1980, «L'historiographie hellénistique et son influence sur Cicéron », Euphrosyne 10,

p. 7-24.

MARCHAL L. 1987, « L'histoire pour Cicéron (I) », LEC 55, p. 41-64.

MÜNZER F. 1927, RE 13, 2, s.v. « L. Lucceius » 6, col. 1554-1559.

PALADINI V. 1947, « Sul pensiero storiografico di Cicerone », Latomus 6, p. 329-344.

RAMBAUD M. 1952, Cicéron et l'histoire romaine [Thèse de doctorat : Lettres : Paris].

TAYLOR L.R. 1949, « On the chronology of Cicero's letters of 55-56 B.C. », CPh 44, 4, p. 217-221.

\section{NOTES}

1. Hartog, Casevitz 1999.

2. Cicéron, de Or. 2, 51-64.

3. Cicéron, ad Fam. 5, 12.

4. Hartog, Casevitz 1999, p. 155.

5. Nous savons par une lettre de Cicéron à Atticus (Att. 4, 13, 2) - que L.-A. Constans (Cicéron, Correspondance, III, $\mathrm{n}^{\circ} 128$ ) et D.R. Shackleton Bailey (Cicero's Letters to Atticus, $\mathrm{n}^{\circ} 87$ ) datent tous deux de novembre 55 - que le de Oratore est achevé à cette date. Le doute subsiste sur la lettre à Lucceius qui aurait été écrite pour les uns en juin 56 (c'est la datation proposée par L.-A. Constans dans la CUF), pour d'autres en avril 55 (Taylor 1949 - suivi par D.R. Shakleton Bailey, Cicero's Letters to his friends, $\left.\mathrm{n}^{\circ} 22\right)$. En tout état de cause, la lettre à Lucceius apparait comme antérieure au de Oratore.

6. Cicéron, de Or. 2, 36 (Historia uero testis temporum, lux ueritatis, uita memoriae, magistra uitae, nuntia uetustatis...) et 2, 62-6 sur les « lois de l'histoire». 
7. Sur cette lecture, voir Rambaud 1952, p. 13 sq.

8. Voir par ex. Paladini 1947.

9. C'est en substance la position de M. Rambaud ; elle est reprise par Marchal 1987, p. 56 : «bien qu'il soit regrettable que, pour se défendre, il <Cicéron> ait recouru au genre historique, nous ne saurions utiliser cette lettre pour critiquer sa conception de l'histoire ».

10. Nous connaissons trois lettres de Cicéron à Lucceius - ad Fam. 5, 12, (CUF, $n^{\circ} 112$ ) ; 5,13 (CUF, $\left.\mathrm{n}^{\circ} 566\right) ; 5,15$ (CUF, $\mathrm{n}^{\circ} 628$ ) - et une lettre de Lucceius à Cicéron, ad Fam. 5, 14 (CUF, $\mathrm{n}^{\circ}$ 624). Mais l'index de la CUF fait apparaître plus de quinze mentions de L. Lucceius, en particulier dans les lettres de Cicéron à Atticus.

11. Sur le personnage, voir Münzer 1927. La notice de F. Münzer peut aujourd'hui être complétée par celle qui vient d'être établie par Benferhat 2005, dans laquelle on trouvera des informations et une bibliographie à jour.

12. La date de 67, qui paraissait assurée à partir de la correction de Dion Cassius, 36, 41, 1-2 (voir Münzer 1927, col.1555), a été remise en question. Pour la discussion, voir Broughton 1986, p. 127-128 : il est possible que le préture de L. Lucceius date seulement de 62. Si l'on maintient 67, cela signifie que L. Lucceius a été préteur un an avant Cicéron. À la suite de sa préture, il aurait refusé la province de Sardaigne.

13. C'est sans doute ce soutien que Cicéron évoque dans une autre de ses lettres à Lucceius, datée de 46 : das enim mihi iucundas recordationes conscientiae nostrae rerumque earum quas te in primis auctore gessimus... (ad Fam. 5, 13, 4).

14. Sur cette candidature, voir Cicéron, Att. 1, 17, 11 (CUF, $n^{\circ} 23$ ) et Att. 2, 1, 9 (CUF, $n^{\circ} 27$ ). Selon Suétone, Caes. 19, c'est Lucceius qui, grâce à sa fortune considérable, avait financé la campagne de César en même temps que la sienne.

15. César, $B C$ 3, 18, 3.

16. Cicéron, ad Fam. 5,14 , datée de mai 45 (CUF, $n^{\circ} 624$ ). Cicéron lui répond peu après : cf. ad Fam. 5, 15 (CUF, $\mathrm{n}^{\circ}$ 628).

17. C'est une hypothèse que n'exclut pas Hinard 1985, p. 488-490.

18. Cicéron, ad Fam. 5, 12, $7:$ :.. auctoritas clarissimi et spectatissimi uiri et in rei publicae maximis grauissimisque causis cogniti atque in primis probati...

19. Hall 1998.

20. Cicéron, Att. 1, 16, 15 (CUF, $\mathrm{n}^{\circ} 22$ ).

21. Cicéron, Att. 2, 1, 2 (CUF, $\mathrm{n}^{\circ} 27$ ).

22. Selon Deniaux 1993, p.519, les liens entre Cicéron et Lucceius restent essentiellement formels.

23. Cicéron souligne en effet la lourdeur de la tâche entreprise par Lucceius : Neque tamen haec cum scribebam eram nescius quantis oneribus premerere susceptarum rerum et iam institutarum (ad Fam. 5, 12, 2).

24. Cicéron, ad Fam. 5, 12, 6: Quo mihi acciderit optatius si in hac sententia fueris, ut a continentibus tuis scriptis, in quibus perpetuam rerum gestarum historiam complecteris... Comme l'a noté Bardon 1952, p. 263-264, l'expression scripta continentia (« un récit continu») employée pour définir l'œuvre de Lucceius et la référence à «la suite ininterrompue des événements historiques » (perpetua rerum gestarum historia) renvoient à un récit de type annalistique. Le terme d'Annales se trouve d'ailleurs au paragraphe précédent, avec une comparaison rappelant l'origine que les Romains attribuaient au genre : Etenim ordo ipse annalium mediocriter nos retinet quasi enumeratione fastorum (ad Fam. 5, 12, 5).

25. Cicéron, ad Fam. 5, 12, 2 : «Mais, puisque je vois que tu as désormais presque achevé l'histoire de la guerre sociale et de la guerre civile, et que tu m'as dit que tu commençais à écrire le reste, je n'ai pas voulu manquer d'attirer ton attention sur la question suivante : préfères-tu traiter ce qui me concerne en le mêlant à la trame des autres événements ou bien, comme l'on fait beaucoup de Grecs - Callisthène pour la guerre de Phocide, Timée pour celle de Pyrrhus, Polybe pour celle de 
Numance, qui tous ont traité les guerres dont je viens de parler à part de leurs histoires continues - toi aussi séparer de la même façon la conjuration qui a touché l'État des guerres que nous avons livrées à l'extérieur contre nos ennemis? » Le verbe seiungere est repris plus loin par secernere (Quo mihi acciderit optatius si in hac sententia fueris, ut a continentibus tuis scriptis, in quibus perpetuam rerum gestarum historiam complecteris, secernas... - ad Fam. 5, 12, 6).

26. Cicéron, ad Fam. 5, 12, 4: «Si je t'amène à entreprendre ce travail, tu trouveras, j'en suis persuadé, un sujet digne de ton talent et de tes facultés. En effet, du début de la conjuration jusqu'à mon retour, il me semble que l'on peut écrire un ouvrage de taille moyenne... »

27. C'est ce que prouve l'exemple de Salluste, qui remonte comme on sait jusqu'à l'arrivée au pouvoir de Sylla dans sa recherche des causes profondes des événements de 63.

28. Cicéron, ad Fam. 5, 12, 2 (cité plus haut). On notera que les historiens grecs sont nommés dans l'ordre chronologique, du plus ancien au plus récent. La guerre de Phocide est la troisième guerre sacrée (356-351), à laquelle Callisthène avait consacré une monographie.

29. Cicéron, ad Fam. 5, 12, 2 (perpetuae suae historiae).

30. Cicéron, ad Fam. 5, 12, 3 : Itaque te plane etiam atque etiam rogo ut et ornes ea uehementius etiam quam fortasse sentis et in eo leges historiae neglegas...

31. Cicéron, ad Fam. 5, 12, 2 (Italici belli et ciuilis historia) et 5, 12, 6 (perpetua rerum gestarum historia).

32. Cicéron, ad Fam. 5, 12, 2 : deesse mihi nolui quin te admonerem ut cogitares coniunctene malles cum reliquis rebus nostra contexere an... L.-A. Constans (Cicéron, Correspondance, II, p. 159) traduit nostra par «l'histoire de mon consulat» - ce qui rend incontestablement l'idée - mais le terme signifie plus prosaïquement « ce qui me concerne ». Il est intéressant de constater que Cicéron s'exprime de la même manière (meae res et non historia) quand il évoque, pour Atticus, sa lettre à Lucceius, dont il est si content (ualde bella est) : Epistulam Lucceio nunc quam misi, qua meas res ut scribat rogo... (Att. 4, 6, 4 = CUF, $\mathrm{n}^{\circ} 113$ ).

33. Cicéron, ad Fam. 5, 12, 6 : «Voilà pourquoi tu exauceras mes vœux si tu décides de séparer du récit continu dans lequel tu embrasses la suite ininterrompue des événements historiques ce que l'on pourrait appeler le drame de ma vie, avec ce que j'ai fait et ce qui m'est arrivé. Il comporte en effet différents actes et de nombreux changements dûs à mes décisions ou aux circonstances. »

34. Au risque de forcer le trait on pourrait presque traduire ici mutationes par «coups de théâtre ".

35. Cicéron, ad Fam. 5, 12, 2: «Et en même temps, si tu portes toute ton attention sur un seul sujet et un seul personnage, je vois déjà avec quelle énergie tu traiteras tout avec plus d'abondance et plus d'éclat ".

36. Le verbe ornare renvoie alors au processus d'«embellissement du discours». Dans la digression sur l'histoire du de Oratore, c'est leur capacité à embellir le discours qui distingue les premiers historiens grecs ou latins - qui ne la possèdent pas (qui neque tenent quibus rebus ornetur oratio - de Or. 2, 53) - des représentants de l'histoire littéraire telle qu'Antoine voudrait la voir pratiquée à Rome et dont Hérodote, qui princeps genus hoc ornauit (de Or. 2, 55), est le premier représentant en Grèce.

37. Voir par ex., Plaute, Epid.194-195. Le verbe ornare - dont il y a quatre occurrences dans la lettre (§ $2 ; \S 3(\mathrm{x} 2) ; \S 6)$ auxquelles on peut ajouter une occurrence d'ornatior (\$ 2) - renvoie, au moins une fois, de façon explicite à un processus contraire aux lois de l'histoire : Itaque te plane etiam atque etiam rogo ut et ornes ea uehementius etiam quam fortasse sentis et in eo leges historiae neglegas... (Cicéron, ad Fam. 5, 12, 3).

38. Voir par ex. Mandel 1980.

39. Le créateur du genre serait Naevius. Nous connaissons grâce à Varron $(L .9,78)$ un court fragment de sa tragédie intitulée Clastidium et consacrée à la bataille du même nom. 
40. Le témoignage d'Asinius Pollion se trouve dans une lettre qu'il a adressée à Cicéron ( $a d$ Fam. 10, 32, 3 = CUF $n^{\circ}$ 915). La tragédie en question avait pour sujet la mission que Cornelius Balbus avait menée en 49 pour le compte de César afin de convaincre (sans résultat) le proconsul L. Lentulus de trahir Pompée.

41. Cicéron, ad Fam. 5, 12, 7 : placet enim Hector ille mihi Naeuianus, qui non tantum "laudari" se laetatur sed addit etiam "a laudato uiro". La même citation se trouve dans une lettre de Cicéron à Caton (ad Fam. 15, 6, 1 = CUF, $\mathrm{n}^{\circ}$ 271). La citation doit provenir de l'Hector proficiscens de Naevius.

42. Cicéron, ad Fam. 5, 12, 4-5: Multam etiam casus nostri uarietatem tibi in scribendo suppeditabunt plenam cuiusdam uoluptatis, quae uehementer animos hominum in legendo te scriptore tenere possit. Nihil est enim aptius ad delectationem lectoris quam temporum uarietates fortunaeque uicissitudines. quae etsi nobis optabiles in experiendo non fuerunt, in legendo tamen erunt iucundae. Habet enim praeteriti doloris secura recordatio delectationem. [...] Etenim ordo ipse annalium mediocriter nos retinet quasi enumeratione fastorum; at uiri saepe excellentis ancipites uariique casus habent admirationem, exspectationem, laetitiam, molestiam, spem, timorem ; si uero exitu notabili concluduntur, expletur animus iucundissima lectionis uoluptate.

43. Polybe, Hist. X, 21, 8. Sur les rapports entre biographie et éloge, voir par ex. Jenkinson 1967.

44. On ne compte pas moins de huit occurrences de laus/laudare: § 2 (Cicéron); §5 (Épaminondas); §6 (Lucceius); § 7 (Agésilas); § 7 (Hector chez Naevius); § 8 (les auteurs d'autobiographie). S’y ajoutent deux occurrences de celebrare - $\$ 1$ (Cicéron) ; § 6 (Cicéron) -, une occurrence de illustrare - $\$ 1$ (Cicéron) - et les occurrences de ornare signalées plus haut. J'ai précisé entre parenthèses le personnage concerné.

45. Cicéron, ad Fam. 5, 12, 3: "C'est pourquoi je te demande clairement encore et encore d'embellir ces faits avec plus de passion même que tu n'en ressens peut-être et de négliger dans cet ouvrage les lois de l'Histoire; quant à cette complaisance sur laquelle tu as écrit de si belle façon dans une préface et dont tu montres qu'elle n'a pas pu davantage te faire changer d'avis que le Plaisir n'a pu le faire pour l'Hercule de Xénophon, si elle me recommande à toi avec trop de passion ne la rejette pas et accorde à notre affection un peu plus même que ce que permettra la vérité. " Le choix qu'avait eu à faire le jeune Hercule entre le Plaisir et la Vertu fait l'objet d'une allégorie que Xénophon rapporte en l'attribuant au sophiste Prodicus (Mém.2, 1, 21); l'épisode est repris par Cicéron, Off. 1, 118.

46. L'œuvre a en effet été écrite, sans doute en 360 ou 359, après la mort d'Agésilas en guise d'éloge funèbre, et non de son vivant.

47. Cicéron, ad Fam. 5, 12, 7 : «Et il ne faut pas moins citer l'illustre Agésilas, le Spartiate - qui n'a pas voulu que les peintres ou les sculpteurs fissent son portrait - que ceux qui se sont démenés pour que cela fût fait. Un seul petit livre de Xénophon a facilement surpassé, quand il s'agissait de louer ce roi, tous les portraits et toutes les statues du monde ».

48. Ces connaissances apparaissent clairement dans la digression sur l'histoire que Cicéron prête à Antoine dans le de Oratore.

49. Nous savons qu'Isocrate - dont Cicéron fait dans le de Or. 2, 57 et dans le Brutus 56, 204, le maître d'Éphore et de Théopompe - avait écrit une biographie à fonction élogieuse d'Évagoras, le roi de Salamine, dans l'île de Chypre, mort assassiné en 374 ; l'Évagoras d'Isocrate précède de quelques années l'Agésilas de Xénophon. Le genre perdure au moins jusqu'à Polybe, qui cite luimême sa biographie en trois livres de Philopœmen (Hist. X, 21).

50. Les parallèles entre l'Agésilas et les livres 3 et 4 des Helléniques sont signalés dans les notes de la traduction que M. Casevitz vient de donner de ce texte: Xénophon, Constitution des Lacédémoniens; Agésilas; Hiéron ; suivi de Pseudo-Xénophon, Constitution des Athéniens, 2008.

51. Cicéron, de Or. 2, 58 : Denique etiam a philosophia profectus princeps Xénophon Socraticus ille...

52. Cicéron, ad Fam. 5, 12, 4 : «En effet, du début de la conjuration jusqu'à mon retour, il me semble que l'on peut écrire un ouvrage de taille moyenne, dans lequel tu pourras faire usage de ta science bien connue des bouleversements politiques, qu'il s'agisse d'exposer les causes des 
révolutions ou les remèdes aux maux, lorsque tu critiqueras ce que tu jugeras blâmable et que tu approuveras, en donnant tes raisons, ce qui aura ta faveur; et si, comme tu en as l'habitude, tu penses devoir traiter la question avec plus de liberté, tu stigmatiseras la perfidie, les embûches, la trahison dont beaucoup ont usé contre moi. De plus mes vicissitudes t'offriront dans l'écriture une grande variété pleine d'une sorte de plaisir, capable, grâce à un écrivain comme toi, de grandement captiver l'esprit des lecteurs. »

53. Cicéron, de Or. 2, 63. Polybe, Hist. X, 21, 3-5, critique aussi les historiens qui font l'histoire des cités, mais ne disent rien de la vie des hommes éminents.

54. Cicéron, ad Fam. 5, 12, 6: «Et quant à moi, je ne suis pas assez fou pour vouloir être recommandé à une gloire éternelle par un homme qui ne serait pas capable, en m'y recommandant, d'obtenir la gloire qui est due à son propre talent. »

55. Tout juste peut-on noter que ce désir d'« immortalité immédiate » est ici parfaitement avoué et assumé dès le début de la lettre (ad Fam. 5, 12,1) par l'orateur.

\section{RÉSUMÉS}

Il ne faut pas sous-estimer l'importance de la lettre à Lucceius dans la réflexion cicéronienne sur l'histoire. Que Cicéron cherche à obtenir de Lucceius une œuvre qui serve à sa propagande, cela va sans dire. Il n'en reste pas moins que sa lettre met en lumière les liens étroits qui existent pour les Anciens, comme en témoigne l'historiographie grecque, entre l'histoire et au moins une certaine forme de biographie, celle qui relève de l'éloge. De ce point de vue, la position exprimée ici par Cicéron - les deux genres obéissent à des lois différentes mais demandent les mêmes qualités de la part de ceux qui voudraient les pratiquer - invite à reconsidérer les questions génériques : elle revient en effet à faire de la biographie peut-être moins un genre littéraire à part que l'une des modalités de l'histoire.

\section{INDEX}

nomsmotscles Asinius Pollion, César, Cicéron, Cornelius Balbus, Dion Cassius, Éphore, Isocrate, Lucceius (Lucius), Naevius, Plaute, Polybe, Suétone, Théopompe, Varron, Xénophon

Keywords : amplification, biography, praise, ethos of the historian, history and tragedy, hellenistic historiography, Roman historiography, literature and politics, monography, objectivity in history, political propaganda, praetexta, historical truth

Index géographique : Sardaigne

Mots-clés : amplification, biographie, éloge, ethos de l'historien, histoire et tragédie, historiographie hellénistique, historiographie romaine, littérature et politique, monographie, objectivité de l'historien, propagande politique, tragédie prétexte, vérité historique

\section{AUTEURS}

\section{GÉRARD SALAMON}

ENS de Lyon, CEROR 